\title{
Four equivalent lot-sizing models
}

\author{
Wilco van den Heuvel *, Albert P.M. Wagelmans \\ Econometric Institute and Erasmus Research Institute of Management, \\ Erasmus University Rotterdam, P.O. Box 1738, 3000 DR Rotterdam, \\ The Netherlands
}

Econometric Institute Report EI 2007-30

May 2007

\begin{abstract}
We study the following lot-sizing models that recently appeared in the literature: a lotsizing model with a remanufacturing option, a lot-sizing model with production time windows, and a lot-sizing model with cumulative capacities. We show the equivalence of these models with a classical model: the lot-sizing model with inventory bounds.

Key words: Lot-sizing; Equivalent models
\end{abstract}

\section{Introduction}

Recently, a number of new lot-sizing models appeared in the literature. In turns out that the models are equivalent to a classical lot-sizing model, the lot-sizing model with bounded inventory (LSB). The three models that turn out to be equivalent with the LSB model are:

1. the lot-sizing model with a remanufacturing option

2. the lot-sizing model with production time windows

3. the lot-sizing model with cumulative capacities.

In this paper we will show the equivalence between the models by reformulating them in a specific form.

The remainder of the paper is organized as follows. In Section 2 we will describe the LSB model, give a mathematical formulation and reformulate this model in the specific form. In Section 3 we will consider the recent models in the order of publication date and discuss the equivalence with the LSB model. In Section 4 we provide an overview of the complexity results for the different models.

\section{Lot-sizing with bounded inventory}

The lot-sizing model with bounded inventory (LSB) can be described as follows (see [5]). Given a finite and discrete time horizon and a deterministic (known) demand in each time period, find the production plan that satisfies the demand and minimizes total cost. The costs include fixed setup cost for each period with positive production, unit production cost for each item produced, and

* Corresponding author.

E-mail address: wvandenheuvel@few.eur.nl (W. van den Heuvel). 
unit holding cost for each item held in stock. So far the problem is equivalent to the classical lotsizing problem of [11]. However, in the LSB model the amount of stock in each period is bounded from below and above. Lower bounds may occur when safety stocks are desired and upper bounds in case of limited warehouse capacity.

Given a problem instance defined by the parameters,

$T$ : time horizon,

$d_{t} \geq 0:$ demand in period $t$,

$l_{t} \geq 0$ : lower bound on ending inventory in period $t$,

$u_{t} \geq 0$ : upper bound on ending inventory in period $t$,

$K_{t}:$ setup cost in period $t$,

$p_{t}$ : unit production cost in period $t$,

$h_{t}$ : unit holding cost in period $t$,

and using the decision variables,

$x_{t}$ : production in period $t$,

$I_{t}$ : ending inventory in period $t$, the problem can be formulated as follows

$$
\begin{array}{llll}
{[\mathrm{LSB}]} & \min & \sum_{t=1}^{T}\left(K_{t} \delta\left(x_{t}\right)+p_{t} x_{t}+h_{t} I_{t}\right) & \\
\text { s.t. } & I_{t}=I_{t-1}+x_{t}-d_{t} & t=1, \ldots, T \\
& l_{t} \leq I_{t} \leq u_{t} & t=1, \ldots, T \\
& x_{t} \geq 0 & t=1, \ldots, T \\
& I_{0}=0, &
\end{array}
$$

where

$$
\delta(z)= \begin{cases}0 & \text { for } z=0 \\ 1 & \text { for } z>0\end{cases}
$$

The objective function minimizes total setup, production and holding cost. The first set of constraints models the inventory balance constraints, the second set ensures that the inventory bounds are not violated, the third set are the non-negativity constraints and w.l.o.g. the initial inventory equals zero. The feasible region in the $(x, I)$ space is determined by the tuple $(d, l, u)$.

In fact, [5] considers a more general model that allows for general concave cost functions, backlogging and negative demands. However, because the other lot-sizing models do not allow for these features, we restrict ourselves to the case above. Love [5] developed an $\mathcal{O}\left(T^{3}\right)$ algorithm for the general model by considering the corresponding network flow problem and exploiting the extreme flow properties of an optimal solution.

To show that three recent models are equivalent to model [LSB], we will reformulate model [LSB] to a specific form. First, note that $I_{t}=\sum_{i=1}^{t}\left(x_{i}-d_{i}\right)$. Substituting this in [LSB] yields

$$
\begin{array}{llll}
{\left[\mathrm{LSB}^{\prime}\right] \quad \min } & \sum_{t=1}^{T}\left(K_{t} \delta\left(x_{t}\right)+p_{t} x_{t}+h_{t} \sum_{i=1}^{t}\left(x_{i}-d_{i}\right)\right) & \\
& \text { s.t. } & \sum_{i=1}^{t} x_{i} \geq l_{t}+\sum_{i=1}^{t} d_{i} & t=1, \ldots, T \\
& \sum_{i=1}^{t} x_{i} \leq \sum_{i=1}^{t} d_{i}+u_{t} & t=1, \ldots, T \\
& x_{t} \geq 0 & t=1, \ldots, T .
\end{array}
$$

Defining $c_{t}=p_{t}+\sum_{i=t}^{T} h_{i}, L_{t}=l_{t}+\sum_{i=1}^{t} d_{i}$ and $U_{t}=\sum_{i=1}^{t} d_{i}+u_{t}$, we get the formulation

$$
\begin{array}{llll}
\text { [LSB"] } \min & \sum_{t=1}^{T}\left(K_{t} \delta\left(x_{t}\right)+c_{t} x_{t}+C\right) & \\
\text { s.t. } & L_{t} \leq \sum_{i=1}^{t} x_{i} \leq U_{t} & t=1, \ldots, T \\
& x_{t} \geq 0 & t=1, \ldots, T
\end{array}
$$

where $C=-\sum_{t=1}^{T} h_{t} \sum_{i=1}^{t} d_{i}$ is a constant. The feasible region for [LSB"] in the $(x)$ space is described by the tuple $(L, U)$.

We may assume w.l.o.g. that the values $L_{t}$ and $U_{t}$ are non-decreasing in $t$. To see that this property holds, consider any feasible solution. For this solution it holds $\sum_{i=1}^{t+1} x_{i} \geq \sum_{i=1}^{t} x_{i} \geq L_{t}$. Now if $L_{t}>L_{t+1}$ for some $t$, then we can construct a new set of lower bounds $L^{\prime}$ where $L_{1}^{\prime}=L_{1}$ 
and $L_{t+1}^{\prime}=\max \left\{L_{t+1}, L_{t}^{\prime}\right\}$ without changing the feasible region. In a similar way it follows that $U_{t}$ can be assumed to be non-decreasing. Namely, for any feasible solution it holds $\sum_{i=1}^{t-1} x_{i} \leq$ $\sum_{i=1}^{t} x_{i} \leq U_{t}$. Therefore, we can define an alternative set of bounds $U_{t}^{\prime}$ where $U_{T}^{\prime}=U_{T}$ and $U_{t-1}^{\prime}=\min \left\{U_{t-1}, U_{t}^{\prime}\right\}$ without changing the feasible region. Clearly, the bounds $L_{t}^{\prime}$ and $U_{t}^{\prime}$ are non-decreasing in $t$.

It follows from the reformulation and the discussion above that there exists a feasible solution if we can find a set of bounds $(L, U)$ satisfying

Property $1 L_{t} \leq U_{t}$ for $t=1, \ldots, T$ and $L_{t}, U_{t}$ are non-decreasing in $t$.

Furthermore, the transformation from model [LSB] to [LSB"] shows that any problem instance $(d, l, u)$ in the $(x, I)$ space can be transformed into an equivalent problem instance $(L, U)$ in the $(x)$ space. The opposite also holds. Given bounds $L_{t}, U_{t}$ satisfying Property 1 , we can construct a problem instance for [LSB] by setting $d_{t}=L_{t}-L_{t-1}$ (with $\left.L_{0} \equiv 0\right), l_{t}=0$ and $u_{t}=U_{t}-L_{t}$. This also implies that we can reformulate a problem instance $(d, l, u)$ as an equivalent problem instance $\left(d^{\prime}, 0, u^{\prime}\right)$.

For simplicity we define the problem instances only by the parameters that describe the feasible region and omit the cost parameters. It will turn out that all lot-sizing models have the same cost structure: a production setup cost, unit production cost and unit holding cost. We will refer to this cost structure as lot-sizing cost.

\section{Three models equivalent to the LSB model}

In the remainder of the paper we will show that the three other lot-sizing models can be formulated as [LSB"] and have bounds $(L, U)$ satisfying Property 1 , which implies they are equivalent to the LSB model. In fact, Property 1 is used by [9] to show the equivalence between the lot-sizing model with a remanufacturing option and the LSB model and by [12] to show the equivalence between the lot-sizing model with production time windows and the LSB model.

\subsection{Lot-sizing with a remanufacturing option}

The lot-sizing problem with a remanufacturing option is an extension of the classical WagnerWhitin model. The additional feature is that in each period a deterministic amount of returned items (returns for short) enters the system. These returns can be remanufactured to satisfy demand besides regular manufacturing. This means that there are two types of inventory: the inventory of returns and the inventory of serviceables, where a serviceable is either a newly manufactured item or a remanufactured returned item.

Richter and Sombrutzki [6] and Richter and Weber [7] consider a special case of this problem where manufacturing is not allowed and where sufficient returns enter the system. We will refer to this model as the LSR model. Using the additional notation

$r_{t} \geq 0$ : amount of returns in period $t$

$K_{t}^{r}$ : remanufacturing setup cost in period $t$,

$p_{t}^{r}$ : unit remanufacturing cost in period $t$,

$h_{t}^{s}$ : unit holding cost for serviceables in period $t$,

$h_{t}^{r}$ : unit holding cost for returns in period $t$,

and using the decision variables

$x_{t}^{r}$ : remanufacturing quantity in period $t$,

$I_{t}^{s}$ : ending inventory of serviceables in period $t$,

$I_{t}^{r}$ : ending inventory of returns in period $t$, the problem can be formulated as 
$[\mathrm{LSR}] \quad \min \sum_{t=1}^{T}\left(K_{t}^{r} \delta\left(x_{t}^{r}\right)+p_{t}^{r} x_{t}+h_{t}^{s} I_{t}^{s}+h_{t}^{r} I_{t}^{r}\right)$

$$
\begin{array}{lll}
\text { s.t. } & I_{t}^{s}=I_{t-1}^{s}+x_{t}^{r}-d_{t} & t=1, \ldots, T \\
& I_{t}^{r}=I_{t-1}^{r}-x_{t}^{r}+r_{t} & t=1, \ldots, T \\
& I_{t}^{s}, I_{t}^{r}, x_{t} \geq 0 & t=1, \ldots, T \\
& I_{0}^{s}=I_{0}^{r}=0 . &
\end{array}
$$

The first set of constraints models the inventory balance of serviceables and the second set models the inventory balance of returns. A problem instance in the $\left(x^{r}, I^{r}, I^{s}\right)$ space is described by the tuple $(d, r)$. Clearly, the problem has a feasible solution if and only if $\sum_{i=1}^{t} r_{i} \geq \sum_{i=1}^{t} d_{i}$ for $t=1, \ldots, T$.

Again we can reformulate the problem by substituting $I_{t}^{s}=\sum_{i=1}^{t}\left(x_{i}^{r}-d_{i}\right)$ and $I_{t}^{r}=\sum_{i=1}^{t}\left(r_{i}-\right.$ $\left.x_{i}^{r}\right)$ in $[\mathrm{LSR}]$ obtaining the model

$$
\begin{array}{rlll}
{\left[\mathrm{LSR}^{\prime}\right]} & \min & \sum_{t=1}^{T}\left(K_{t}^{r} \delta\left(x_{t}^{r}\right)+p_{t}^{r} x_{t}+h_{t}^{s} \sum_{i=1}^{t}\left(x_{t}^{r}-d_{t}\right)+h_{t}^{r} \sum_{i=1}^{t}\left(r_{i}-x_{i}^{r}\right)\right) & \\
\text { s.t. } & \sum_{i=1}^{t} x_{i}^{r} \geq \sum_{i=1}^{t} d_{i} & t=1, \ldots, T \\
& \sum_{i=1}^{t} x_{i}^{r} \leq \sum_{i=1}^{t} r_{i} & t=1, \ldots, T \\
& x_{t}^{r} \geq 0 & t=1, \ldots, T .
\end{array}
$$

Clearly, model [LSR'] is equivalent to [LSB"] by letting $L_{t}=\sum_{i=1}^{t} d_{i}, U_{t}=\sum_{i=1}^{t} r_{i}$ and $c_{t}=$ $p_{t}^{r}+\sum_{i=t}^{T}\left(h_{i}^{s}-h_{i}^{r}\right)$, and noting that $L_{t}$ and $U_{t}$ satisfy Property 1 for a feasible problem instance. If $h_{i}^{s}<h_{i}^{r}$ the cost parameter $c_{t}$ may be negative. In this case it may be optimal to have a positive ending inventory of serviceables, i.e., $I_{T}^{s}>0$.

The network flow representation of the LSR problem (see Figure 1) leads a simple representation of the properties of an optimal solution for the LSB model described in [5]. To this end, consider again model [LSB]. A period $t$ is called a production period if there is a positive amount of production in period $t$, i.e., $x_{t}>0$. A period $t$ is called an inventory period if the ending inventory in period $t$ exactly equals the lower or upper bound, i.e., $I_{t}=l_{t}$ or $I_{t}=u_{t}$. Love [5] shows that there exists an optimal solution satisfying the following properties:

- between any two inventory periods there is at most one production period,

- between any two production periods there is at least one inventory period.

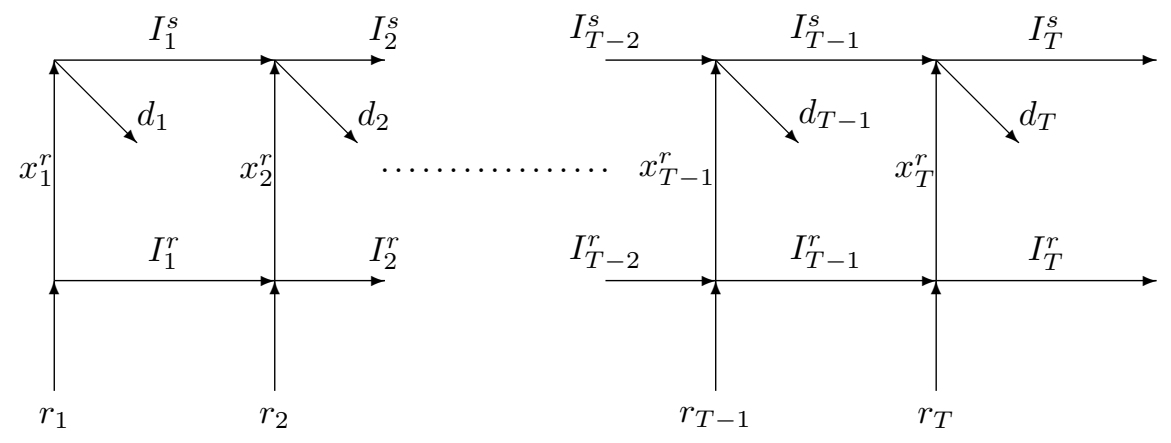

Figure 1: Network flow representation of the LSR model

Now return to the LSR model. We call period $t$ a remanufacturing period if $x_{t}^{r}>0$. Consider a feasible solution of problem LSR represented by a graph consisting of the arcs that have a positive flow in the network formulation. It is well known that an optimal solution can be found among the extreme flows in the network, which means that the corresponding graph cannot have any cycles (see Figure 2). 


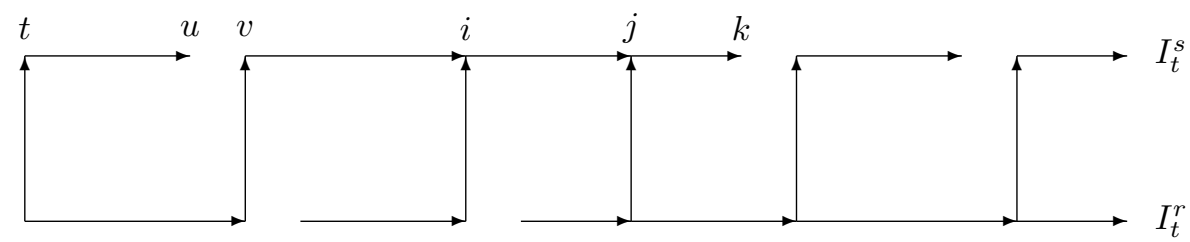

Figure 2: Graph corresponding to an extreme flow solution for the LSR problem

Because of the no cycle property, it follows (see Figure 2) that between any two consecutive remanufacturing periods $t$ and $v$, there must be a period $u, t \leq u<v$, with $I_{t}^{s}=0$ or $I_{t}^{r}=0$. Noting that remanufacturing in the LSR model corresponds to production in the LSB model, this means there exists a period $t$ with $I_{t}=l_{t}$ or $I_{t}=u_{t}$ in the $(x, I)$ space. Furthermore, between any two consecutive periods $i$ and $k$ satisfying $I_{i}^{\alpha}=0$ and $I_{k}^{\beta}=0$ with $\alpha, \beta \in\{r, s\}$, there is at most one period with $x_{j}^{r}>0$. In the $(x, I)$ space this means that there is at most one production period between any two inventory periods. These are exactly the optimality properties described by [5] (which are derived from another network flow representation).

\subsection{Lot-sizing with production time windows}

The lot-sizing model with production time windows (LSP) (see [12]) is probably most surprisingly equivalent with the LSB model. In this lot-sizing model there are orders $k=1, \ldots, N$ where each order has a demand $D_{k}$ that must be satisfied in period $e_{k}$ by producing in some period $t \geq b_{k}$ with $1 \leq b_{k} \leq e_{k} \leq T$. Interval $\left[b_{k}, e_{k}\right]$ is called the production time window associated with order $D_{k}$. Notice that this problem is different from the lot-sizing problem with delivery time windows, where every demand needs to be satisfied within a time window (see [3]).

Two different models are considered: the distinct order case and the indistinguishable orders case. To see the difference between the two cases, consider a 2-orders problem with $D_{1}$ and $D_{2}$, $D_{1}<D_{2}$, and with time windows $\left[b_{1}, e_{1}\right]$ and $\left[b_{2}, e_{2}\right]$ satisfying $b_{2}<b_{1} \leq e_{1}<e_{2}$. So time window 1 is strictly included in time window 2 . Now producing $D_{1}$ units in period $b_{2}$ and $D_{2}$ units in period $e_{2}$ is an infeasible solution for the distinct order case but a feasible solution for the indistinguishable orders case. Wolsey [12] shows that the LSP model with indistinguishable orders and the LSP model with distinct orders and non-inclusive time windows are equivalent to the LSB model. For completeness of the paper we also show this equivalence.

First, we will consider the model with indistinguishable orders. Define

$a_{t}=\sum_{\left\{k: b_{k}=t\right\}} D_{k}$ : the production quantity that becomes available in period $t$,

$d_{t}=\sum_{\left\{k: e_{k}=t\right\}} D_{k}$ : the amount of demand that needs to be satisfied in period $t$.

Following [12] and letting $I_{t}^{2}$ be the (artificial) 'stock of available production' in period $t$ with zero holding cost, the problem can be formulated as

$$
\begin{array}{llll}
\text { [LSPI] } \min & \sum_{t=1}^{T}\left(K_{t} \delta\left(x_{t}\right)+p_{t} x_{t}+h_{t} I_{t}\right) & \\
& \text { s.t. } & I_{t}=I_{t-1}+x_{t}-d_{t} & t=1, \ldots, T \\
& I_{t}^{2}=I_{t-1}^{2}-x_{t}+a_{t} & t=1, \ldots, T \\
& I_{t}, I_{t}^{2}, x_{t} \geq 0 & t=1, \ldots, T \\
& I_{0}=I_{0}^{2}=0 . &
\end{array}
$$

Note that the feasible region is described by the same type of constraints as model [LSR]. Therefore, by setting

$$
\begin{aligned}
& L_{t}=\sum_{i=1}^{t} d_{i}=\sum_{\left\{k: e_{k} \leq t\right\}} D_{k}: \text { the amount of demand that needs to be satisfied up to period } t, \\
& U_{t}=\sum_{i=1}^{t} a_{i}=\sum_{\left\{k: b_{k} \leq t\right\}} D_{k}: \text { the amount of available production up to period } t,
\end{aligned}
$$


we get a formulation equivalent to [LSB"]. Note that $L_{t}$ and $U_{t}$ are non-increasing, $L_{t} \leq U_{t}$ as $b_{k} \leq e_{k}$ and, hence, satisfy Property 1 .

Now consider the LSP model with distinct orders. Introducing the variable $z_{t, k}$, the amount produced for order $k$ in period $t, k=1, \ldots, N$ and $b_{k} \leq t \leq e_{k}$, the mathematical model becomes

$$
\begin{array}{lll}
\text { [LSPD] } \min & \sum_{t=1}^{T}\left(K_{t} \delta\left(x_{t}\right)+p_{t} x_{t}+h_{t} I_{t}\right) & \\
\text { s.t. } & I_{t}=I_{t-1}+x_{t}-d_{t} & t=1, \ldots, T \\
& \sum_{t=b_{k}}^{e_{k}} z_{t, k}=D_{k} & k=1, \ldots, N \\
& x_{t}=\sum_{\left\{k: b_{k} \leq t \leq e_{k}\right\}} z_{t, k} & t=1, \ldots, T \\
& I_{t}, x_{t}, z_{t, k} \geq 0 & t=1, \ldots, T, k=1, \ldots, N \\
& I_{0}=0 . &
\end{array}
$$

The $x_{t}$ variables in model [LSPD] satisfy

$$
\sum_{i=1}^{t} x_{i}=\sum_{i=1}^{t} \sum_{\left\{k: b_{k} \leq i \leq e_{k}\right\}} z_{i, k} \geq \sum_{\left\{k: e_{k} \leq t\right\}} D_{k}=L_{t}
$$

and

$$
\sum_{i=1}^{t} x_{i}=\sum_{i=1}^{t} \sum_{\left\{k: b_{k} \leq i \leq e_{k}\right\}} z_{i, k} \leq \sum_{\left\{k: b_{k} \geq t\right\}} D_{k}=U_{t} .
$$

Therefore, any feasible solution $(x)$ for model [LSPD] is feasible for [LSPI]. Note that the reverse does not hold in general. However, the reverse claim holds in the case of non-inclusive time windows. Wolsey [12] shows that for this case the following two properties hold:

\section{Property 2}

(i) A set of non-inclusive time windows can be ordered such that for $k=1, \ldots, N-1$ either $b_{k}<b_{k+1}$ and $e_{k} \geq e_{k+1}$, or $b_{k}=b_{k+1}$ and $e_{k}<e_{k+1}$.

(ii) With non-inclusive time windows ordered as in (i), any feasible solution has the property (or can be modified such) that order $k$ is produced before or at the same time as order $k+1$ for $k=1, \ldots, N-1$.

(Note that we slightly modified Property (ii) of [12].) Property (ii) implies that any feasible solution $(x)$ for [LSPI] can be transformed into a feasible solution for the [LSPD] model with non-inclusive time windows. To see that Property (ii) holds for a feasible $(x)$ of [LSPI], we can assign production to orders starting with $x_{1}$ and $D_{1}$. Then, when arriving at some period $t$ and order $k$, production from period $t+1$ is assigned to order $k$ if all production from period $t$ has been assigned and production from $t$ is assigned to order $k+1$ if order $k$ is fulfilled. For a different proof we refer to [12].

We remark that the bounds $(L, U)$ for the lot-sizing model with production time windows satisfy $L_{T}=U_{T}$. Hence, any feasible solution will have zero inventory in period $T$. This may not be the case for an optimal solution to model [LSB"] with $L_{T}<U_{T}$. (Note that we made no restrictions on the signs of $K_{t}$ and $c_{t}$.) If $L_{T}<U_{T}$ and $c_{t}<0$ for some $t$, then there may be an optimal solution with $L_{T}<\sum_{i=1}^{T} x_{i} \leq U_{T}$ and hence $I_{T}=\sum_{i=1}^{T} x_{i}-L_{T}>0$. However, if one prefers a problem instance with $L_{T}=U_{T}$, then adding a dummy period $T+1$ with $U_{T+1}=L_{T+1}=U_{T}$ and $K_{T+1}=c_{T+1}=0$ does not change the feasible region and leads to the desired problem instance.

\subsection{Lot-sizing with cumulative capacities}

The last model equivalent to the LSB problem is the lot-sizing model with cumulative capacities (LSC) (see [8]). In this lot-sizing problem each period has a production capacity, but unused capacity is transferred to the next period. This may be the case when capacity is not perishable, such as raw material or money. This is in contrast to the case of perishable capacity, such as time. 
Sargut and Romeijn [8] show that the problem is NP-hard for general non-decreasing production and holding cost functions and develop a polynomial time approximation scheme for non-increasing cost functions.

Furthermore, the authors consider the problem with concave cost functions. Using the additional notation

$C_{t}$ : (non-perishable) production capacity in period $t$,

$C_{1, t}=\sum_{i=1}^{t} C_{i}$ : cumulative production capacity up to period $t$,

Sargut and Romeijn [8] formulate the problem as follows (we changed the objective function to a lot-sizing cost structure instead of concave functions).

$$
\begin{array}{lll}
\text { [LSC] } \min & \sum_{t=1}^{T}\left(K_{t} \delta\left(x_{t}\right)+p_{t} x_{t}+h_{t} I_{t}\right) & \\
\text { s.t. } & I_{t}=I_{t-1}+x_{t}-d_{t} & t=1, \ldots, T \\
& \sum_{i=1}^{t} x_{i} \leq C_{1, t} & t=1, \ldots, T \\
& x_{t}, I_{t} \geq 0 & t=1, \ldots, T \\
& I_{0}=0, &
\end{array}
$$

Using a similar approach as before, the problem can be rewritten as

$$
\begin{array}{llll}
{\left[\mathrm{LSC}^{\prime}\right] \quad \min } & \sum_{t=1}^{T}\left(K_{t} \delta\left(x_{t}\right)+c_{t} x_{t}+C\right) & \\
& \text { s.t. } & \sum_{i=1}^{t} x_{i} \geq \sum_{i=1}^{t} d_{i} & t=1, \ldots, T \\
& & \sum_{i=1}^{t} x_{i} \leq C_{1, t} & t=1, \ldots, T \\
& & x_{t} \geq 0 & t=1, \ldots, T,
\end{array}
$$

Clearly, by setting $L_{t}=\sum_{i=1}^{t} d_{i}$ and $U_{t}=C_{1, t}$, again we have a model equivalent to [LSB"]. Furthermore, the bounds $(L, U)$ satisfy Property 1 for any feasible problem instance of [LSC'].

\section{Complexity results}

In this section we summarize some complexity results for the 'different' problems. As already mentioned, [5] developed an $\mathcal{O}\left(T^{3}\right)$ time algorithm for the LSB model with concave cost functions. Recently, [4] considered the LSB model with lot-sizing cost and, using a similar approach as [10], developed an $\mathcal{O}\left(T^{2}\right)$ time algorithm for general cost parameters and an $\mathcal{O}(T)$ algorithm for the case of non-speculative motives (also called Wagner-Whitin cost), i.e., $c_{t} \geq c_{t+1}$ for $t=1, \ldots, T-1$ in model [LSB"].

Furthermore, [4] proved that in the case of non-speculative motives there exists an optimal solution where production occurs if inventory equals its lower bound, generalizing the zero-inventory ordering property. However, as shown in Section 2, every problem instance $(d, l, u)$ can be rewritten as an instance with lower bounds equal to zero, i.e., $\left(d^{\prime}, 0, u^{\prime}\right)$. Hence, the result of [4] confirms the well-known result that the LSB model with no lower bounds satisfies the zero-inventory ordering property in case of non-speculative motives.

The LSR model has been considered by [6], [7] and [9]. Richter and Sombrutzki [6] and Richter and Weber [7] notice that the model can be transformed into a lot-sizing model with inventory bounds and develop an $\mathcal{O}\left(T^{2}\right)$ Wagner-Whitin type algorithm for the case of non-speculative (NS) motives. However, they do not show that the reverse also holds. Van den Heuvel [9] shows the equivalence between the LSR and LSB model and develops an $\mathcal{O}\left(T^{2} \log T\right)$ algorithm for the case with general lot-sizing cost parameters. It follows from the discussion above that both complexity results for the LSR model can be improved by applying the algorithms for the LSB model.

The LSP model has been considered by [12]. He developed an $\mathcal{O}(T N)$ algorithm for the case of non-inclusive time windows and for the case of indistinguishable orders, where $N$ is the number of orders. As $N \leq 2 T-1$ for these cases, the algorithm runs in $\mathcal{O}\left(T^{2}\right)$ time. This means that [12] and [4] developed an $\mathcal{O}\left(T^{2}\right)$ for the LSB model at the same time. Interestingly, the approaches used are quite different. Again we mention that [12] showed the equivalence between the LSP and LSB model. 


\begin{tabular}{lllll}
\hline Author(s) & Problem & Cost structure & Complexity & Comments \\
\hline$[5]$ & LSB & Concave & $\mathcal{O}\left(T^{3}\right)$ & \\
{$[4]$} & LSB & Lot-sizing & $\mathcal{O}\left(T^{2}\right)$ & \\
{$[4]$} & LSB & Lot-sizing & $\mathcal{O}(T)$ & NS motives \\
{$[6]$ and $[7]$} & LSR & Lot-sizing & $\mathcal{O}\left(T^{2}\right)$ & NS motives \\
{$[9]$} & LSR & Lot-sizing & $\mathcal{O}\left(T^{2} \log T\right)$ & \\
{$[12]$} & LSP & Lot-sizing & $\mathcal{O}\left(T^{2}\right)$ & \\
{$[8]$} & LSC & Concave & $\mathcal{O}\left(T^{4}\right)$ & \\
{$[2]$} & LSB & Lot-sizing with fixed & $\mathcal{O}\left(T^{2}\right)$ & \\
{$[12]$} & LSP & holding cost component & & \\
\hline
\end{tabular}

Table 1: Complexity results

Finally, the LSC model has been considered by [8]. Besides developing a polynomial time approximation scheme for the case of general non-increasing cost functions, they developed an $\mathcal{O}\left(T^{4}\right)$ algorithm for the case of concave cost functions. It follows that the latter result can be improved by applying the algorithm of [5].

We end this section with some remarks on the complexity of some closely related problems. Atamtürk and Küçükyavuz [1] consider the LSB model where the holding cost functions are setuplinear, which is more general than the classical lot-sizing cost assumption. In [2] they show that this problem can be solved in $\mathcal{O}\left(T^{2}\right)$. Hence, this algorithm outperforms the other $\mathcal{O}\left(T^{2}\right)$ algorithms in the sense that their algorithm solves a more general problem in the same running time.

Wolsey [12] considers another generalization of the LSB model with time-invariant production capacities in each period, i.e., $x_{t} \leq C$ for $t=1, \ldots, T$ with $C$ the production capacity. He shows that this problem can be solved in $\mathcal{O}\left(T^{4}\right)$ time. This means that all models in this paper with lot-sizing cost and constant capacities can be solved in $\mathcal{O}\left(T^{4}\right)$ time. We end the paper with a summary of the complexity results in Table 1. 


\section{References}

[1] A. Atamtürk and S. Küçükyavuz. Lot sizing with inventory bounds and fixed costs: Polyhedral study and computation, Operations Research 53 (2005) 711-730.

[2] A. Atamtürk and S. Küçükyavuz. A note on "Lot sizing with inventory bounds and fixed costs: Polyhedral study and computation": An $O\left(n^{2}\right)$ algorithm for lot sizing with inventory bounds and fixed costs, (2006). (Unpublished manuscript)

[3] C.-Y. Lee, S. Çetinkaya, and A.P.M. Wagelmans. A dynamic lot size model with demand time windows, Management Science 47 (2001) 1384-1395.

[4] T. Liu. Economic lot sizing problem with inventory bounds, European Journal of Operational Research (2006). (to appear)

[5] F. Love. Bounded production and inventory models with piecewise concave costs, Management Science 20 (1973) 313-318.

[6] K. Richter and M. Sombrutzki. Remanufacturing planning for the reverse Wagner/Whitin models, European Journal of Operational Research 121 (2000) 304-315.

[7] K. Richter and J. Weber. The reverse Wagner/Whitin model with variable manufacturing and remanufacturing cost, International Journal of Production Economics 71 (2001) 447-456.

[8] F.Z. Sargut and H.E. Romeijn. Lot-sizing with non-stationary cumulative capacities. Operations Research Letters 35 (2007) 549-557.

[9] W. van den Heuvel. The economic lot-sizing problem: New results and extensions, PhD thesis, Erasmus University Rotterdam, 2006.

[10] A.P.M. Wagelmans, C.P.M. van Hoesel, and A. Kolen. Economic lot sizing: An $\mathrm{O}(n \log n)$ algorithm that runs in linear time in the Wagner-Whitin case, Operations Research 40 (1992) S145-S156.

[11] H.M. Wagner and T.M. Whitin. Dynamic version of the economic lot size model, Management Science 5 (1958) 89-96.

[12] L.A. Wolsey. Lot-sizing with production and delivery time windows, Mathematical Programming, Series A 107 (2006) 471-489. 